\title{
Spectroscopy at the High-Energy Electron Beam Ion Trap (SuperEBIT)
}

Klaus Widmann

Peter Beiersdorfer

Jose R. Crespo López-Urrutia

Steve Elliott

Prepared for Submittal to

Proceedings of the 2nd Euroconference on Atomic Physics

with Stored Highly Charged Ions

June 2-7, 1996, Stockholm, Sweden

July 10, 1996

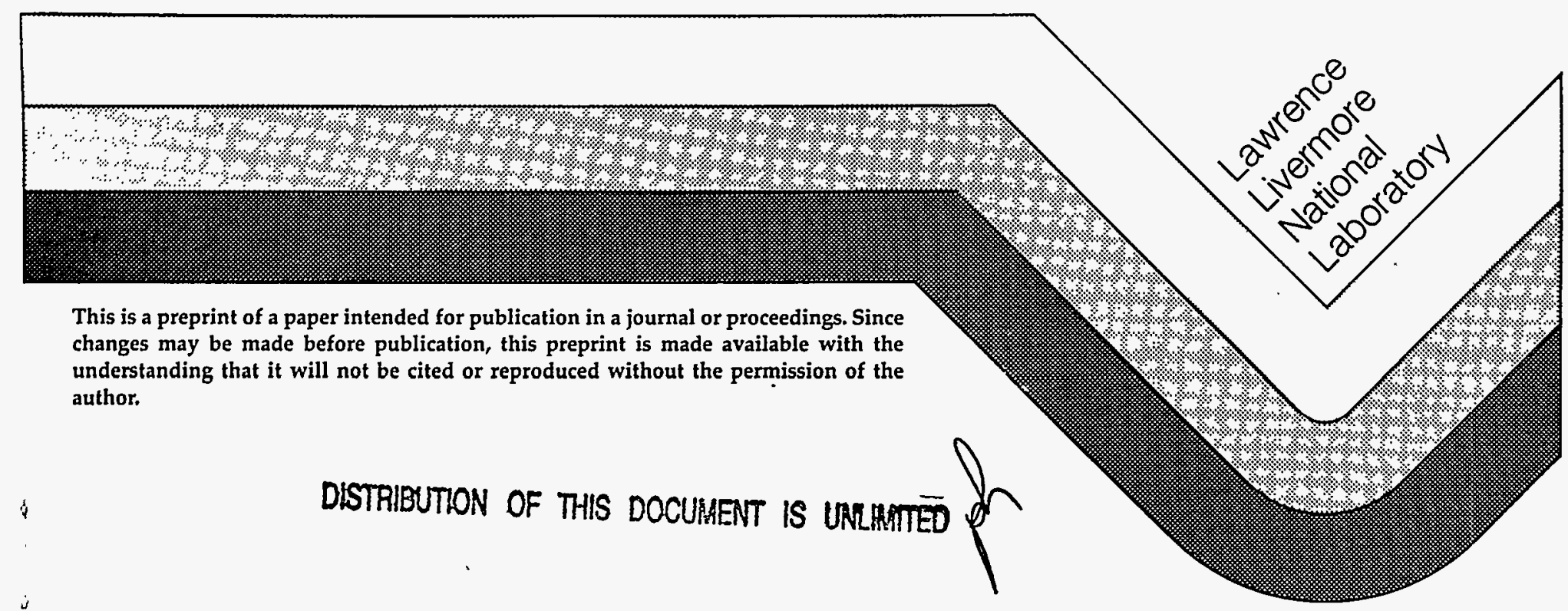




\section{DISCLAIMER}

This document was prepared as an account of work sponsored by an agency of the United States Government. Neither the United States Government nor the University of California nor any of their employees, makes any warranty, express or implied, or assumes any legal liability or responsibility for the accuracy, completeness, or usefulness of any information, apparatus, product, or process disclosed, or represents that its use would not infringe privately owned rights. Reference herein to any specific commercial products, process, or service by trade name, trademark, manufacturer, or otherwise, does not necessarily constitute or imply its endorsement, recommendation, or favoring by the United States Government or the University of California. The views and opinions of authors expressed herein do not necessarily state or reflect those of the United States Government or the University of California, and shall not be used for advertising or product endorsement purposes. 


\section{DISCLAIMER}

Portions of this document may be illegible in electronic image products. Images are produced from the best available original document. 


\title{
Spectroscopy at the High-Energy Electron Beam Ion Trap (SuperEBIT)
}

\author{
Klaus Widmann ${ }^{1,2}$, Peter Beiersdorfer ${ }^{1}$, José R. Crespo López \\ URRUTiA $^{1}$, AND STEVE R. ELLIOTT ${ }^{3}$ \\ ${ }^{1}$ Lawrence Livermore National Laboratory, P.O.Box 808, Livermore CA 94551, USA \\ ${ }^{2}$ Institut für Experimentalphysik, Technische Universität Graz, A-8010 Graz, \\ AUSTRIA \\ ${ }^{3}$ Department of Physics, University of Washington, P.O.Box 351560, Seattle, \\ WA 98195-1560, USA
}

The following progress report presents some of the $x$-ray measurements performed during the last year on the Livermore SuperEBIT facility. The measurements include: direct observation of the spontaneous emission of the hyperfine transition in ground state hydrogenlike holmium, ${ }^{165} \mathrm{Ho}^{66+}$; measurements of the $\mathrm{n}=2 \rightarrow 2$ transition energies in neonlike thorium, $\mathrm{Th}^{80+}$, through lithiumlike thorium, $\mathrm{Th}^{87+}$, testing the predictions of quantum electrodynamical contributions in high- $\mathrm{Z}$ ions up to the $0.4 \%$ level; measurements of the isotope shift of the $\mathrm{n}=2 \rightarrow 2$ transition energies between lithiumlike through carbonlike uranium, ${ }^{233} \mathrm{U}^{89+\ldots .86+}$ and ${ }^{238} \mathrm{U}^{89+\ldots 86+}$, infering the variation of the mean-square nuclear charge radius; and highresolution measurements of the $\mathrm{K} \alpha$ radiation of heliumlike xenon, $\mathrm{Xe}^{52+}$, using a transmission-type crystal spectrometer, resolving for the first time the $1 s 2 p^{3} P_{1} \rightarrow 1 s^{2}{ }^{1} S_{0}$ and $1 s 2 s^{3} S_{1} \rightarrow 1 s^{21} S_{0}$ transitions individually. 


\section{Introduction}

The electron beam ion trap (EBIT) facility at the Lawrence Livermore National Laboratory was especially designed for the study of atomic processes of highly charged ions[1]. The high-energy version, dubbed SuperEBIT, operates with an electron beam energy of up to $215 \mathrm{keV}$. Thus, SuperEBIT can produce and trap highly charged ions up to bare uranium, $\mathrm{U}^{92+}[2]$. The ions in the trap are relatively cold, just a few eV per charge[3], and are essentially at rest, which makes SuperEBIT an ideal source for spectroscopic diagnostic of few electron high-Z ions. A detailed description of SuperEBIT may be found elsewhere[4].

The SuperEBIT facility provides different options for injecting ions or neutrals into the trap region. One of the techniques to inject ions into SuperEBIT is based on a metal vapor vacuum arc (MeVVA)[5]. Depending on the operation mode, the MeVVA plasma contains atoms and ions from either the cathode or the trigger material. The ions are extracted from this plasma and focused into the trap region. A method to introduce neutrals into SuperEBIT is a gas injector. The pressure in the gas injector can be adjusted between $10^{-9}$ to $10^{-6}$ Torr, which is at least two to five orders of magnitude higher than the pressure in the trap itself. Precise adjustment of the gas injector ensures that the atoms or molecules intersect the electron beam, where they get ionized and trapped. A continuous injection of low- $\mathrm{Z}$ elements, such as nitrogen or neon, is very important for the evaporative cooling of the collisionally heated highly charged ions[6]. Recently, a technique has been developed which allows to inject materials which are available only in trace quantities[7]. This technique works by plating a small amount of the desired material on the pointed end of a wire probe. The wire probe is positioned near the compressed electron beam and material is removed from the sample by sputtering caused by trapped ions surrounding the electron beam. It enables to run experiments on SuperEBIT with only nanograms of feed material.

The ionization balance in SuperEBIT is optimized by choosing an appropriate elec- 
tron beam energy, trap depth, cooling gas pressure, and the time during which the ions are kept in the trap before they are dumped and the trap is filled with "new" ions. The emission of the trapped ions is observed perpendicular to the electron beam. The confinement of the ions, along a 2-cm-long region within the 70- $\mu$ m-diameter electron beam, makes SuperEBIT a perfect line source, which can be imaged by a spectrometer without applying an additional entrance slit.

During the last year the main research goal of the EBIT spectroscopy group was to extend the spectral range of our high-resolution measurements. With our flatcrystal low-energy and von Hámos-type medium-energy crystal spectrometer we have performed high-resolution measurements between $650 \mathrm{eV}$ and $13000 \mathrm{eV}[8,9,10,11]$. Adding a prism spectrograph and a transmission-type crystal spectrometer to the current EBIT instrumentation made the visible and near UV range, namely $1.5 \mathrm{eV}$ to $6 \mathrm{eV}$, and the hard $\mathrm{x}$-ray regime, beyond $13 \mathrm{keV}$, accessible to high-resolution spectroscopy.

In the following, we review our recent measurements in ascending order regarding to the energy of the observed photons. In Section 2 we describe the direct observation of the hyperfine transition in hydrogenlike holmium, ${ }^{165} \mathrm{Ho}^{66+}$, which is situated in the visible range, i.e. around $2 \mathrm{eV}$. Section 3 contains investigations of $4-\mathrm{keV} \mathrm{x}$ rays and is divided into two parts: The first part presents the precision measurements of the $\mathrm{n}=2 \rightarrow 2$ transition energies in neonlike thorium, $\mathrm{Th}^{80+}$, through lithiumlike thorium, $\mathrm{Th}^{87+}$, and a detailed comparison between the experimental values and theoretical predictions is given for berylliumlike thorium, $\mathrm{Th}^{86+}$. The second part gives a short description of isotope shift measurements of the $\mathrm{n}=2 \rightarrow 2$ transition energies comparing lithiumlike through carbonlike ${ }^{233} \mathrm{U}$ and ${ }^{238} \mathrm{U}$. High-resolution measurements of hard $\mathrm{x}$-rays, around $30 \mathrm{keV}$, are the topic of Section 4 . In particular, it describes the implementation of a transmission-type crystal spectrometer at SuperEBIT and shows measurements of some $\mathrm{n}=2 \rightarrow 1$ transitions of heliumlike xenon, $\mathrm{Xe}^{52+}$. 
2 Visible regime: Direct observation of the hyperfine transition of hydrogenlike holmium, ${ }^{165} \mathrm{Ho}^{66+}$

Using a prism spectrograph we measured the $F=4$ to $F=3$ hyperfine transition of the 1 s ground level of hydrogenlike ${ }^{165} \mathrm{Ho}^{66+}[12]$. The experimental setup is shown in Fig. 1. It consists of a spectrograph with an aperture of $f / 9$, and a cryogenically cooled CCD camera $(1024 \times 1024$ pixels of $24 \mu \mathrm{m}$ side length). The spectral range covered in a single image was from $4175 \AA$ to $6900 \AA$. The beam is stigmatically imaged by two achromats and, thus, spatially resolved in the vertical direction ( $0.1 \mathrm{~mm}$ resolution) allowing to distinguish between line radiation emitted by cooling gas atoms and by trapped ions. Radiation from the cooling gas is only emitted from the region where the atomic or molecular beam from the gas injector crosses the electron beam, whereas emission from trapped ions can be observed along the whole height of the trap. The spatial resolution is, therefore, important to determine the nature of the light emitting species. The ability to distinguish between atomic and ionic emission lines was also useful for the wavelength calibration of the optical spectra. In particular, we used lines emitted from neutral neon to calibrate the wavelength scale of our spectra.

A MeVVA with a holmium cathode was used to inject holmium ions into SuperEBIT. Measurements with and without injecting holmium were done consecutively throughout the whole experiment in order to eliminate the background, e.g. thermal radiation from the heated electron-gun filament, as well as emission from cooling-gas atoms. To determine the ionic species and charge balance in SuperEBIT, we monitored the radiative recombination $(R R)$ lines, produced when electrons from the beam are captured into open shells of the ions, with a high-purity germanium detector (see Fig. 1). A RR-spectrum with a holmium-filled trap, at an electron-beam energy of $\approx 133 \mathrm{keV}$ and an electron-beam current of $285 \mathrm{~mA}$, is shown in Fig. 2 (a). In this case the holmium charge balance was: $0.5 \%$ bare $\mathrm{Ho}^{67+}, 6 \%$ hydrogenlike $\mathrm{Ho}^{66+}, 40 \%$ heliumlike $\mathrm{Ho}^{65+}, 25 \%$ lithiumlike $\mathrm{Ho}^{64+}$, and $28.5 \%$ lower charge states. 
The result of the measurements are shown in Fig. 2 (b), which displays two background-corrected spectra. The first was obtained when the trap was filled with hydrogenlike holmium, $\mathrm{Ho}^{66+}$, and clearly shows a single feature at $5726.4 \pm 1.5 \AA$. The second spectrum was obtained by repeating the experiment with a much lower electron-beam energy, namely $63 \mathrm{keV}$, to avoid production of $\mathrm{Ho}^{66+}$ but still keeping a similar abundance regarding the heliumlike and lower charge states. The fact that this spectrum does not show any indication of any line proves that hydrogenlike holmium accounts for the observed line. Our experimental result for the $F=4$ to $F=3$ hyperfine transition of the 1s ground level of hydrogenlike ${ }^{165} \mathrm{Ho}^{66+}$ is $5726.4 \pm 1.5 \AA$ (air), which corresponds to a vacuum wavelength of $5727.9 \pm 1.5 \AA$, and a transition energy of $2.1646(6) \mathrm{eV}$, respectively[12].

Figure 3 shows a comparison between our result and the values predicted by theory. Using Shabaev's formula[13] for the energy difference between two neighboring levels in a hydrogenlike ion $2.199 \mathrm{eV}$ can be derived. this value has an uncertainty of at least $15 \mathrm{meV}$ due to the uncertainty in the value for nuclear magnetic moment of ${ }^{165} \mathrm{Ho}, \mu_{I}=$ 4.173(27) $\mu_{N}$, tabulated by Lederer and Shirley[14], and omits QED corrections. Our measured value significantly disagrees with this prediction. Based on recently published calculations regarding the two dominant QED corrections $[15,16]$, we estimate for $Z=67$ a 9.4-meV contribution due to the vacuum polarization[15] and a $-19.3-\mathrm{meV}$ shift for the self-energy correction[16]. But even the QED-corrected value, i.e., $2.189 \mathrm{eV}$ disagrees with our measurement by $24 \mathrm{meV}$. The weak point of the calculation appears to be the nuclear magnetic moment. The value given by Lederer and Shirley was measured using the atomic beam resonance method[17], later recalculated[18], and is cited in the literature as $4.160(27) \mu_{N}$ or $4.173(27) \mu_{N}$, depending on the diamagnetic correction employed. A newer measurement was performed by Nachtsheim[19] with five times higher accuracy, and the result, $4.132(5) \mu_{N}$, is compiled in the reviews of Peker[20]. Using this value the theoretical value, including the QED corrections, for the 
hyperfine transition energy is $2.1675(28) \mathrm{eV}$. This differs from our measured value of $2.1646(6) \mathrm{eV}$ by only $0.13 \%$ and shows agreement within the $1-\sigma$ confidence level. Our measurement, thus, easily distinguishes between the two values of the nuclear magnetic moment, and clearly favors the one measured by Nachtsheim[19].

\section{Low-energy $x$ rays: Fine structure of $\mathrm{L}-$ shell transitions}

For few-electron high-Z ions the $\mathrm{n}=2$ intrashell transitions are in the low-energy $\mathrm{x}$ ray regime. The effects of QED are enhanced in the spectra of the heaviest elements, because QED contributions scale with higher power of $\mathrm{Z}$ than the Dirac energies. For example, the Lamb shift for the $2 \mathrm{~s}$ level in uranium is on the order of $-70 \mathrm{eV}$ of which about $-40 \mathrm{eV}$ are from QED contributions. Thus, the high $-\mathrm{Z}$ elements provide an ideal platform for sensitive tests of QED theory in intense nuclear fields. Additionally, the simplified atomic structure of few-electron high-Z systems allows to interpret the result of isotope shift measurements in terms of the variation in the mean nuclear radius.

\subsection{Structure and Lamb shift of $L$-shell transitions in few-electron high-Z ions}

High-precision measurements of the $2 p_{3 / 2} \rightarrow 2 s_{1 / 2}$ transitions have been performed for lithiumlike through neonlike thorium[21] at the SuperEBIT facility. These transitions are around $4 \mathrm{keV}$ for the thorium ions. The spectra were taken with a von Hámostype crystal spectrometer, sketched in Fig. 4 . The crystal, a $\operatorname{LiF}(200)$ with $2 \mathrm{~d}=4.027$ $\AA$, had a radius of curvature of $30 \mathrm{~cm}$. The diffracted $\mathrm{x}$ rays were recorded with a position-sensitive proportional counter with a $10.0 \times 3.0 \times 0.4 \mathrm{~cm}^{3}$ active volume, filled with $70 \%$ xenon and $30 \%$ methane, 1.2 atm overpressure. The resolving power of this setup was $\mathrm{E} / \Delta \mathrm{E} \approx 2200$ and was mainly limited by $270-\mu \mathrm{m}$ spatial resolution of the proportional counter. As in the case of the ${ }^{165} \mathrm{Ho}^{66+}$ measurement, the instrumentation 
included a high-purity germanium detector, which was used to provide a survey of the $\mathrm{x}$-ray emission and, thus, of the charge balance in SuperEBIT. The information about the charge balance was used to support the determination of the ionic species emitting the lines observed with the crystal spectrometer. High-resolution spectra of the $2 p_{3 / 2} \rightarrow 2 s_{1 / 2}$ transitions in the eight charge states from $\mathrm{Th}^{80+}$ to $\mathrm{Th}^{87+}$ are shown in Fig. 5. These spectra were taken under different ionization-balance conditions. Figure 5(a) represents a higher abundance of higher charge states than Fig. 5(b). The spectra were calibrated using transitions in hydrogenlike argon, $\mathrm{Ar}^{17+}$, namely $\mathrm{Ly}-$ $\beta, L y-\gamma$, and $L y-\delta$. The uncertainty for the determination of the thorium transition energies is $\pm 0.13 \mathrm{eV}$, i.e., about $30 \mathrm{ppm}$, for the strong lines and 0.20 to $0.43 \mathrm{eV}$, i.e., 45 to $100 \mathrm{ppm}$, for the weak lines. The accuracy of our measurements allows to test the QED contributions, which are predicted to be around $-35 \mathrm{eV}$ for the highly charged thorium ions, within $0.4 \%$.

Stimulated by earlier measurements of the same transitons in lithiumlike through neonlike uranium[22], also performed at the SuperEBIT facility, a lot of effort has been put in calculating the level scheme for these few-electron high- $Z$ ions. Figure 6 shows a comparison between our measured values of the transition energies and several theoretical values for the berylliumlike thorium, $\mathrm{Th}^{+86}$, and uranium, $\mathrm{U}^{88+}$. In our comparison we include: multiconfiguration Dirac-Fock (MCDF) calculations, manybody perturbation theory (MBPT) calculations, and the relativistic configurationinteraction (CI) method. For the MCDF approach the code of Grant and co-workers was used and the QED corrections were taken from calculations in the extendedaverage level scheme[23, 24]. The result, $4071.7 \mathrm{eV}$ for $\mathrm{Th}^{86+}$ and $4505.3 \mathrm{eV}$ for $\mathrm{U}^{88+}$, significantly disagrees with our measured values, i.e., 4068.47(13) eV, and 4501.72(27), respectively[22, 21]. The MBPT calculations seem to produce better predictions than the MCDF method. In particular, we refer to two recently published papers[25, 26]. The first one[25] combines MBPT calculations of the atomic structure[27] with $a b$ ini- 
tio QED corrections[28, 29, 30]. To give a measure of the convergence of the MBPT method two different model potentials were used in this work to start the MBPT calculations, which were carried out to second order and include a quadrupole-shaped nuclear charge distribution[31]. The formalism was applied to highly charged uranium ions and, thus, two values for the berylliumlike $\mathrm{U}^{88+}$ are given, namely $4502.88 \mathrm{eV}$ and $4504.28 \mathrm{eV}$. These calculated values, still, disagree with our measurements. The second MBPT result[26] we have included in Fig. 6 also has been carried out to second order and includes a QED estimate based on the one-electron Lamb shift data calculated in a $\left(1 \mathrm{~s}^{2}\right)$ potential[29]. The theoretical values for the transition energies obtained by applying spherical nuclear potentials are $4069.02 \mathrm{eV}$ for $\mathrm{Th}^{86+}$ and 4501.60 $\mathrm{eV}$ for $\mathrm{U}^{88+}$. In the case of berylliumlike uranium, the calculated and measured values agree but there remains significant disagreement for $\mathrm{Th}^{86+}$. Taking the same theoretical method but including a non-spherical charge distribution for the nucleus leads to $4068.36 \mathrm{eV}$ for the $\mathrm{Th}^{86+}$ transition energy, which, now, agrees with our experimentally obtained value. The third theoretical approach, presented in Fig. 6, to obtain the $2 p_{3 / 2} \rightarrow 2 s_{1 / 2}$ transitions energies combines CI energies with $a b$ initio QED and mass polarization corrections[32]. The CI results for $\mathrm{U}^{88+}$ calculated with the $\mathrm{N}$-electron Dirac-Slater(DS) and two-electron Dirac-Hartree(DH) potentials are $4501.53 \mathrm{eV}$ (CI$\mathrm{DS})$ and $4501.61 \mathrm{eV} .(\mathrm{CI}-\mathrm{DH})$, respectively. These provide excellent agreement with our measurement of $4501.72(27) \mathrm{eV}$.

\subsection{Isotope shift measurements}

The $2 p_{3 / 2} \rightarrow 2 s_{1 / 2}$ transition energies in lithiumlike through carbonlike uranium, $\mathrm{U}^{89+}$ to $\mathrm{U}^{86+}$, have been measured for two isotopes, ${ }^{233} \mathrm{U}$ and ${ }^{238} \mathrm{U}[33]$. It was the first measurement to isolate the variation of nuclear effects in the $\mathrm{x}$-ray transitions of fewelectron heavy ions. The experimental setup, shown in Fig. 4, was similar to the one described in Section 3.1. For this measurement the radius of curvature of the $\operatorname{LiF}(200)$ 
crystal was $75 \mathrm{~cm}$. The resolving power of the von Hámos-type crystal spectrometer was approximately 5000 . The radioactive ${ }^{23} \mathrm{U}$ ions were introduced into the trap using a thin platinum wire. The tip of the wire was plated with a total mass of 100 $\mathrm{ng}$ of ${ }^{233} \mathrm{U}$, and less than $10 \mathrm{ng}$ were consumed during the course of the experiment. A MeVVA source containing a ${ }^{238} \mathrm{U}$ cathode provided the ${ }^{238} \mathrm{U}$ ions. Data collection alternated between ${ }^{233} \mathrm{U}$ and ${ }^{238} \mathrm{U}$ spectra. Figure 7 shows the aquired spectra. Each spectrum represents $150 \mathrm{~h}$ of data accumulation at a electron-beam energy of $135 \mathrm{keV}$ and electron-beam current of $180-220 \mathrm{~mA}$. The dispersion of the spectrometer was determined using previous ${ }^{238} \mathrm{U}$ transition energy measurements[22]. In order to infer the Coulomb shift $\left(\delta E_{\text {Coul }}\right)$ and thus the variation in the mean-square nuclear charge radius $\left(\delta\left\langle r^{2}\right\rangle\right)$, the isotopic variation of the specific mass shift, of the QED terms, and of the nuclear polarization has to be estimated[34, 27, 28, 35, 36]. The uncertainty in this estimation is, however, small, which makes the use of highly charged ions ideal for measurements of the isotopic variation of the nuclear charge radii.

The measurements for the energy shift, $\Delta \mathrm{E}$, are listed in Table 1 together with the infered values for $\delta E_{\text {Coul }}$ and $\delta\left\langle r^{2}\right\rangle$. The average value which can be derived for $\delta\left\langle r^{2}\right\rangle^{233,238}$ is $-0.457(43) \mathrm{fm}^{2}$. This result can be compared with that of previous studies: neutral-atom optical isotope shift measurements obtaining a value of $-0.383(44)$ $\mathrm{fm}^{2}[37,38]$, and muonic-atom $\mathrm{x}$-ray observations achieving $-0.520(81) \mathrm{fm}^{2}$ for the variation of the mean-square nuclear charge radius[31]. The SuperEBIT measurement, thus, favors neither of the earlier measurements. It was the first time that precise isotope shift measurements have been performed in electronic transitions that are strongly affected by QED. The precision of our method rivals that of the "standard" methods allowing a reexamination of much of the nuclear radii data. 


\section{High-energy $\mathrm{x}$-rays: Transmission-type crystal spectrometer}

High-resolution x-ray measurements on EBIT have so far been performed only with reflection-type crystal spectrometers. These have worked efficiently for $\mathrm{x}$-ray energies up to $13 \mathrm{keV}$, e.g., the $\mathrm{K}$-shell radiation of heliumlike krypton[11]. In order to extend crystal spectrometer measurements to higher-energy $\mathrm{x}$ rays from higher- $\mathrm{Z}$ elements, we have designed a transmission-type crystal spectrometer[39]. It enabled us to observe individual transitions in the $\mathrm{K}$-shell spectrum of high- $\mathrm{Z}$ ions well above krypton. In particular, we observed the $1 s 2 p^{3} P_{1} \rightarrow 1 s^{2}{ }^{1} S_{0}$ and $1 s 2 s^{3} S_{1} \rightarrow 1 s^{21} S_{0}$ transitions in heliumlike xenon, $\mathrm{Xe}^{52+}$, which have an energy of about $30 \mathrm{keV}$ and which had never before been spectroscopically resolved. Precision measurements of heliumlike and hydrogenlike xenon $\mathrm{K} \alpha$ transitions have been performed by Briand et al.[40] using a germanium detector with a resolution of $270 \mathrm{eV}$. The current setup improves the spectral resolution by more than an order of magnitude.

Our transmission-type spectrometer design is based on the DuMond geometry[41] employing a cylindrically bent crystal. The radius of curvature of the crystal is the diameter of the so-called Rowland circle (see Fig. 8). Placing the $x$-ray source, i.e., the trap region of SuperEBIT, on the Rowland circle strongly reduces the bandwidth of the diffracted $\mathrm{x}$ rays with the advantage that the throughput is tremendously increased at a given wavelength of interest. If the opening angle of the crystal is small enough, the diffracted $\mathrm{x}$ rays are quasimonochromatic. Therefore, no position sensitive detector is necessary, and solid state detectors, such as high-purity germanium detectors, can be used with almost $100 \%$ counting efficiency for $30 \mathrm{keV}$ photons. To obtain a spectrum we rotate the crystal using a stepper motor mounted on a modified rotation stage. At each crystal position we count the number of photons reaching the detector and tag this number with the current position of the stepper motor. We normalized each step using the countrate of directly excited lines, emitted by the highly charged ions in the trap, which were measured with a second germanium detector. 
A layout of the transmission-type crystal spectrometer for SuperEBIT is shown in Fig. 8. In order to observe the $\mathrm{K} \alpha$ radiation of heliumlike xenon, $\mathrm{Xe}^{52+}$, which is

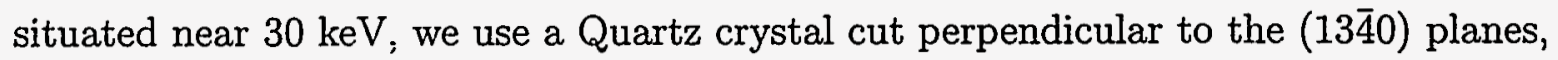
$2 d=2.3604 \AA$. Thus, the nominal Bragg angle is around $\theta=9.9^{\circ}$. The change in the Bragg angle is $0.000741^{\circ} \pm 0.000001^{\circ}$ per step which is equivalent to an energy change of about $2.2 \mathrm{eV}$ for the diffracted photons at these $\mathrm{x}$-ray energies. The radius of curvature of the crystal is $R_{c}=(2713.8 \pm 3.2) \mathrm{mm}$ and was measured using an optical setup. The illuminated area of the crystal is $(60 \times 40) \mathrm{mm}^{2}$. Positioning the crystal so that the electron beam is part of the Rowland circle is non trivial. At perfect alignment the energy spread of the diffracted $x$-ray photons reaching the detector is less than $0.5 \mathrm{eV}$ across the detector area. If the distance between the crystal and SuperEBIT is off by $\pm 10 \mathrm{~mm}$, the bandwidth of the diffracted $\mathrm{x}$ rays is increased to $\approx 3.5 \mathrm{eV}$. The influence of the finite width of the source, i.e., the width of the electron beam $\Delta x_{\text {beam }}=60 \mu \mathrm{m}$, is about $4 \mathrm{eV}$ for every diffracted $\mathrm{x}$-ray photon. Therefore, the nominal resolution of our transmission-type spectrometer cannot be better than $\Delta E=4.5 \mathrm{eV}$, and the resolving power not better than $E / \Delta E=6800$ for this setup. Further limitation of the resolving power is due to the quality of the focus of the bent crystal.

Figure 9 presents the result of the high-resolution measurement of some heliumlike $\mathrm{K} \alpha$ transitions using the transmission-type crystal spectrometer. The spectrum shows the $1 s 2 s^{3} S_{1} \rightarrow 1 s^{2}{ }^{1} S_{0}$, and $1 s 2 p^{3} P_{1} \rightarrow 1 s^{21} S_{0}$, transitions in heliumlike $\mathrm{Xe}^{52+}$. It took 131 hours to collect the amount of counts shown in Fig. 9. One channel represents the sum of the counts obtained during three consecutive steps. Thus, the dispersion is 6.6 $\mathrm{eV}$ per channel. Using a simple Gaussian fit we obtain a full width at half maximum of $(20 \pm 5) \mathrm{eV}$ for these transitions. Therefore, the measured resolving power of our transmission-type crystal spectrometer is about 1500. This shows that the resolving power is limited by the quality of the focus of the crystal. The measurement clearly 
shows the feasibility of resolving individual lines in the spectra of very highly charged ions and sets the stage for further such measurements in the future.

\section{Conclusion}

The spectroscopy measurements at SuperEBIT have been developed to a point where high-precision measurements are possible over a wide range of photon energy, from the visible light to hard $x$ rays. This includes very detailed tests of relativistic $Q E D$ theory in few-electron high-Z systems, i.e., in intense nuclear fields, where theory is tested at the sub $1 \%$ level. SuperEBIT measurements not only test atomic theory but are also sensitive to nuclear parameters. We are able to measure nuclear magnetic momenta at an accuracy level of $0.2 \%$. Moreover, we have been able to measure the isotope variations in the nuclear charge distribution. the measurements are competitive with results obtained with nuclear physics techniques. This is a new area for spectroscopy, and more detailed investigations will be done in the future.

\section{Acknowledgements}

We would like to thank Gregory Brown, Vincent Decaux, and Daniel Savin for their participation on all the experiments. We also thank Ed Magee and Dan Nelson for their technical support. This work was performed under the auspices of U.S.DOE by Lawrence Livermore National Laboratory under contract \# W-7405-ENG-48 and was supported by the Office of Basic Energy Sciences, Division of Chemical Science.

\section{References}

[1] M.A. Levine, R.E. Marrs, D.A. Knapp, and M.B. Schneider, The electron beam ion trap: a new instrument for atomic physics measurements, Phys. Scr. T22 (1988) 
$157-163$.

[2] R.E. Marrs, S.R. Elliott, and D.A. Knapp, Production and trapping of hydrogenlike and bare uranium ions in an electron beam ion trap, Phys. Rev. Lett. 72 (1994) 4082-4085.

[3] P. Beiersdorfer, V. Decaux, K. Widmann, Measurement of the temperature of cold highly charged ions produced in an electron beam ion trap, Nucl. Instrum. Methods B98 (1995) 566-568.

[4] D.A. Knapp, R.E. Marrs, S.R. Elliott, E.W. Magee, and R. Zasadzinski, A highenergy electron beam ion trap for production of high-charge high-Z ions, Nucl. Instrum. Methods A334 (1993) 305-312.

[5] I.G. Brown, J.E. Galvin, R.A. MacGill, and R.T. Wright, Miniature high current metal ion source, Appl. Phys. Lett. 49, (1986) 1019-1021.

[6] B.M. Penetrante, J.N. Bardsley, M.A. Levine, D.A. Knapp, and R.E. Marrs, Evaporative cooling of highly charged dysprosium ions in an enhanced electron beam ion trap, Phys. Rev. A 43 (1991) 4873-4882.

[7] S.R. Elliott, and R.E. Marrs, A wire probe as an ion source for an electron beam ion trap, Nucl. Instrum. Methods B100 (1995) 529-535.

[8] P. Beiersdorfer, and B.J. Wargelin, Low energy $x$-ray spectrometer for an electron beam ion trap, Rev. Sci. Instrum. 65 (1994) 13-17.

[9] P. Beiersdorfer, R.E. Marrs, J.R. Henderson, D.A. Knapp, M.A. Levine, D.B.

- Platt, M.B. Schneider, D.A. Vogel, and K.L. Wong, High-resolution x-ray spectrometer for an electron beam ion trap, Rev. Sci. Instrum. 61 (1990) 2338-2342.

[10] D. Vogel, P. Beiersdorfer, V. Decaux, and K. Widmann, High-pressure positionsensitive proportional counter, Rev. Sci. Instrum. 66 (1995) 776-778.

[11] K. Widmann, P. Beiersdorfer, V. Decaux, and M. Bitter, Measurements of the K $\alpha$ transition energies of heliumlike krypton, Phys. Rev. A 53 (1996) 2200-2205.

[12] J.R. Crespo López-Urrutia, P. Beiersdorfer, D. Savin, and K. Widmann, Direct 
observation of the spontaneous emission of the hyperfine transition $F=4$ to $F=3$ in ground state hydrogen-like ${ }^{165} \mathrm{Ho}^{66+}$ in an electron beam ion trap, submitted to Phys. Rev. Lett.

[13] V.M. Shabaev, Hyperfine structure of hydrogen-like ions, J. Phys. B 27 (1994) $5825-5832$.

[14] E.Browne, J.M. Dairiki, R.E. Doebler, A.A. Shihab-Eldin, L.J. Jardine, J.K. Tuli, A.B. Buyrn Table of Isotopes ed. by C.M. Lederer and V.S. Shirley, seventh editon, Wiley, New York, 1978.

[15] S.M. Schneider, W. Greiner, and G. Soff, Vacuum-polarization contribution to the hyperfine-structure splitting of hydrogenlike atoms, Phys. Rev. A 50 (1994) $118-122$.

[16] H. Persson, S.M. Schneider, W. Greiner, G. Soff, and I. Lindgren, Self-energy correction to the hyperfine structure splitting of hydrogenlike atoms, Phys. Rev. Lett. 76 (1996) 1433-1436.

[17] R.A. Haberstroh, T.I. Moran, and S. Penselin, Direct measurement of the nuclear magnetic dipole moment of $\mathrm{Ho}^{165}$ with the atomic beam magnetic resonance method, Z. Phys. 252 (1972) 421-427.

[18] W. Dankwort and J. Ferch, Reevaluation of the atomic $g_{J}$-factor and the nuclear $g_{I}$-factor of ${ }^{165} \mathrm{Ho}$, Z. Phys. 267 (1974) $239-241$.

[19] G. Nachtsheim, Präzisionsmessung der Hyperfeinstruktur-Wechselwirkung von ${ }^{165}$ Ho im Grundzustand, Ph.D. Thesis, Universität Bonn, 1980, unpublished.

[20] L.K. Peker, Nuclear Data Sheets for $A=165$, Nucl. Data Sheets 50 (1987) 137-227.

[21] P. Beiersdorfer, A. Osterheld, S.R. Elliott, M.H. Chen, D. Knapp, and K. Reed, Structure and lamb shift of $2 p_{3 / 2}-2 s_{1 / 2}$ levels in lithiumlike $\mathrm{Th}^{87+}$ through neonlike $\mathrm{Th}^{80+}$, Phys. Rev. A 52 (1995) 2693-2706. .

[22] P. Beiersdorfer, D. Knapp, R.E. Marrs, S.R. Elliott, and M.H. Chen, Structure and lamb shift of $2 p_{3 / 2}-2 s_{1 / 2}$ levels in lithiumlike $\mathrm{U}^{89+}$ through neonlike $\mathrm{U}^{82+}$, 
Phys. Rev. Lett. 71 (1993) 3939-3942.

[23] I.P. Grant, B.J. McKenzie, P.H. Norrington, D.F. Mayers, and N.C. Pyper, An atomic multiconfigurational Dirac-Fock package, Comput. Phys. Commun. 21 (1980) 207-231.

[24] K.G. Dyall, I.P. Grant, C.T. Johnson, F.A. Parpia, and E.P. Plummer, GRASP a general-purpose relativistic atomic structure program, Comput. Phys. Commun. 55 (1989) 425-456.

[25] W.R. Johnson, J. Sapirstein, and K.T. Cheng, Theory of $2 s_{1 / 2}-2 p_{3 / 2}$ transitions in highly-ionized uranium, Phys. Rev. A 51 (1995) 297-302.

[26] M.S. Safronova, W.R. Johnson, and U.I Safronova, Relativistic many-body calculations of the energies of $n=2$ states for the berylliumlike isoelectronic sequence, Phys. Rev. A 53 (1996) 4036-4053.

[27] S.A. Blundell, W.R. Johnson, and J. Sapirstein, Improved many-body perturbation-theory calculations of the $n=2$ states of lithiumlike uranium, Phys. Rev. A 41 (1990) 1698-1700.

[28] S.A. Blundell, Accurate screened QED calculations in high-Z many-electron ions, Phys. Rev. A 46 (1992) 3762-3775.

[29] K.T. Cheng, W.R. Johnson, and J. Sapirstein, Lamb-shift calculations for noncoulomb potentials, Phys. Rev. A 47 (1993) 1817-1823.

[30] I. Lindgren, H. Persson, S. Salomonson, and A. Ynnerman, Bound-state selfenergy calculation using partial-wave renormalization, Phys. Rev. A 47 (1993) R4555-R4558.

[31] J.D. Zumbro, E.B. Shera, Y. Tanaka, C.E. Bemis Jr., R.A. Nauman, M.V. Hoehn, W. Reuter, and R.M. Steffen, E2 and E4 deformations in ${ }^{233,234,235,238}$ U, Phys. Rev. Lett. 53 (1984) 1888-1892.

[32] K.T. Cheng and M.H. Chen, Relativistic configuration-interaction calculations for the $2 s_{1 / 2}-2 p_{3 / 2}$ transition energies of uranium ions, Phys. Rev. A 53 (1996) 
2206-2210.

[33] S.R. Elliott, P. Beiersdorfer, and M.H. Chen, Trapped-ion technique for measuring the nuclear charge radii of highly charged radioactive isotopes, Phys. Rev. Lett. 76 (1996) 1031-1034.

[34] W.H. King, Isotope shifts in atomic spectra, Plenum Press, New York, 1984.

[35] G. Plunien, B. Muller, W. Greiner, G. Soff, Nuclear polarization in heavy atoms and superheavy quasiatoms, Phys. Rev. A 43 (1991) 5853-5866.

[36] G. Plunien and G. Soff, Nuclear-polarization contribution to the Lamb shift in actinide nuclei, Phys. Rev. A 51 (1995) 1119-1131.

[37] A. Anastassov, Yu.P. Gangrsky, K.P. Marinova, B.N. Markov, B.K. Kul'djanov, and S.G. Zemlyanoi, Nuclear charge radii changes of uranium and hafnium isotopes determined by laser spectroscopy, Hyperfine Interact. 74 (1992) 31-40.

[38] R.T. Brockmeier, F. Boehm, and E.N. Hatch, Observation of the isotope shift of $K_{\alpha 1} \times$ rays of uranium, Phys. Rev. Lett. 15 (1965) 132-135.

[39] K. Widmann, P. Beiersdorfer, G.V. Brown, J.R. Crespo López Urrutia, V. Decaux, D.W. Savin, A high-resolution transmission-type x-ray spectrometer designed for observation of the $\mathrm{K} \alpha$ transitions of highly charged high-Z ions, submitted to Rev. Sci. Instrum. (May 1996).

[40] J.P. Briand, P. Indelicato, A. Simionovici, V. San Vicente, D. Liesen, and D. Dietrich, Spectroscopic study of hydrogenlike and heliumlike xenon ions, Europhys. Lett. 9 (1989) 225-230.

[41] J.W.M. DuMond, A high resolving power, curved-crystal focusing spectrometer for short wave-length x-rays and gamma-rays, Rev. Sci. Instrum. 18 (1947) 626638. 
Table 1: Measured energy shifts between the ${ }^{233} U$ and ${ }^{238} \mathrm{U}$ ions for the transitions shown in Fig. 7 , and infered values for the Coulomb shift $\left(\delta E_{\text {Coul }}\right)$ and the variation in the mean-square nuclear charge radius $\left(\delta\left\langle r^{2}\right\rangle^{233,238}\right)$

\begin{tabular}{lccc}
\hline \hline Key & $\begin{array}{c}E_{233 \mathrm{U}}-E_{238 \mathrm{U}} \\
\mathrm{meV}\end{array}$ & $\begin{array}{c}\delta E_{\text {Coul }} \\
\mathrm{meV}\end{array}$ & $\begin{array}{c}\delta\left\langle r^{2}\right\rangle^{233,238} \\
\mathrm{fm}^{2}\end{array}$ \\
\hline $\mathrm{Li}$ & $256 \pm 118$ & $280 \pm 118$ & $-0.364 \pm 0.153$ \\
$\mathrm{Be}$ & $300 \pm 61$ & $324 \pm 61$ & $-0.436 \pm 0.081$ \\
$\mathrm{~B}-1,2$ & $320 \pm 52$ & $344 \pm 52$ & $-0.455 \pm 0.068$ \\
$\mathrm{C}$ & $362 \pm 62$ & $386 \pm 62$ & $-0.515 \pm 0.083$ \\
\hline \hline
\end{tabular}


Figure 1: Layout of the prism spectrograph in the horizontal plane of EBIT. The electron beam is perpendicular to the page.

Figure 2: (a): Radiative recombination spectrum from SuperEBIT with a holmiumfilled trap showing the presence of the bare $\mathrm{Ho}^{67+}$ and hydrogenic $\mathrm{Ho}^{66+}$ ions in the trap. The electron-beam energy was set to $133 \mathrm{keV}$. (b): Spectra, recorded with the CCD camera, showing the $F=4$ to $F=3$ hyperfine transition of the 1s ground level of hydrogenlike ${ }^{165} \mathrm{Ho}^{66+}$ and the result when there is only heliumlike and lower charge states of holmium in the trap.

Figure 3: Comparison of our measured value for the $F=4$ to $F=3$ hyperfine transition and theoretical predictions. The hatched area marks the 1- $\sigma$ confidence limit of our measurement. Open symbols ( $\triangle$ without, and $\bigcirc$ with QED contributions) refer to calculated values using the nuclear magnetic moment $\left(\mu_{I}\right)$ tabulated by Lederer and Shirley[14]. The --value is based on the same calculations as $\bigcirc$ but using a more recent and much more accurate measurement for $\mu_{I}$ of ${ }^{165} \mathrm{Ho}[19]$. The error bars reflect the uncertainty of the $\mu_{I}$ measurements.

Figure 4: Schematic layout of the van Hámos-type crystal spectrometer on SuperEBIT. A high-purity germanium detector concurrently monitors the $x$-ray emission. The wire probe was used to introduce radioactive ${ }^{233} \mathrm{U}$ ions into the trap (see Section 3.2). The electron beam is perpendicular to the page.

Figure 5: Spectra obtained with the van Hámos-type crystal spectrometer of the $2 p_{3 / 2} \rightarrow 2 s_{1 / 2}$ transitions in lithiumlike $\mathrm{Th}^{87+}$ through neoenlike $\mathrm{Th}^{80+}$. Spectrum (a) was taken at a higher ionization balance conditions than spectrum (b), which favors the lower charge states, i.e. carbonlike through neonlike. The labels show the isoelectronic sequence. If more lines are affiliated to the same ionic species, they are numbered in respect to their transition energies. 
Figure 6: Comparison between our measurment and calculated values for the $\left(2 s_{1 / 2} 2 p_{3 / 2}\right)_{J=1} \rightarrow\left(2 s^{2}\right)_{J=0}$ transition in berylliumlike $\mathrm{Th}^{86+}$ and $\mathrm{U}^{88+}$. The figure consists of two graphs, one for $E_{\text {theo }}>E_{\text {expt }}$, which is on top of the graph representing the case $E_{\text {theo }}<E_{\text {expt }}$. The hatched area refers to the $68 \%$ confidence limit of our measured values. The dashed lines show the energies obtained by theory when neglecting QED contributions. MCDF'93 (+)...multiconfiguration Dirac-Fock calculations[22, 21]. MBPT'95 $(\diamond)$...many-body perturbation theory calculations[25]. The two different results are obtained by starting the calculation with different model potentials. MBPT'96 (口)... Ref.[26]. The open symbols mark calculations using a spherical nuclear charge distribution, the values marked by filled symbols were obtained after feeding a non-spherical nuclear potential into the formalism. CI'96 $(o, x) \ldots$ relativistic configuration interaction calculations with the N-electron Dirac-Slater (DS) and twoelectron Dirac-Hartree (DH) potentials, respectively[32].

Figure 7: Crystal-spectrometer spectra of the $2 p_{3 / 2} \rightarrow 2 s_{1 / 2}$ transitions in lithiumlike $\mathrm{U}^{89+}$ through oxygenlike $\mathrm{U}^{85+}$ for the two isotopes ${ }^{233} \mathrm{U}$ and ${ }^{238} \mathrm{U}$. The labels show the isoelectronic sequence. The ${ }^{233} \mathrm{U}$ spectrum is offset by 500 counts/channel. The dashed lines indicate the center of gravity of the ${ }^{238} \mathrm{U}$ lines.

Figure 8: Layout of the transmission-type crystal spectrometer in the horizontal plane of SuperEBIT. The electron beam is perpendicular to the page. GeSE and GeX are high-purity germanium detectors; $\theta \ldots$ Bragg angle;

Figure 9: First resolved spectrum of the $1 s 2 p^{3} P_{1} \rightarrow 1 s^{21} S_{0}$ and $1 s 2 s^{3} S_{1} \rightarrow 1 s^{21} S_{0}$ transitions in heliumlike $\mathrm{Xe}^{52+}$ observed with the transmission-type crystal spectrometer. 


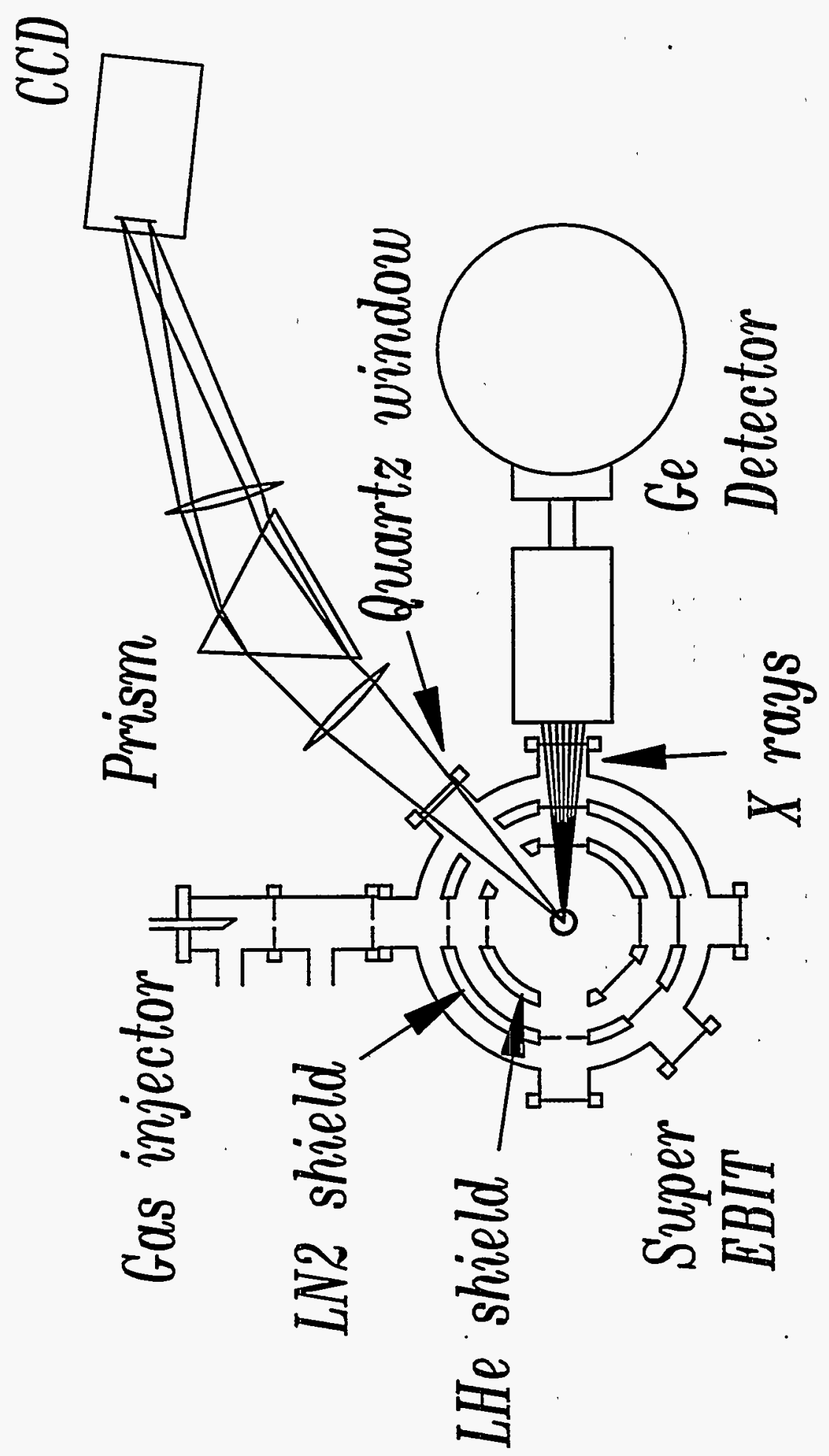

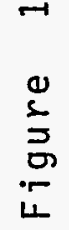



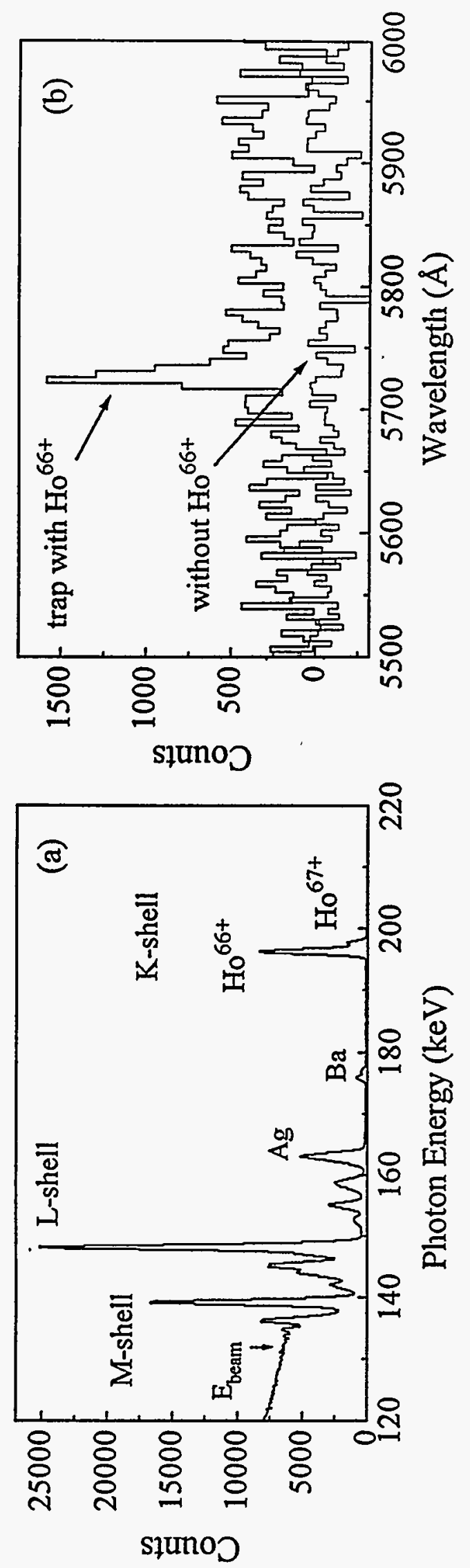


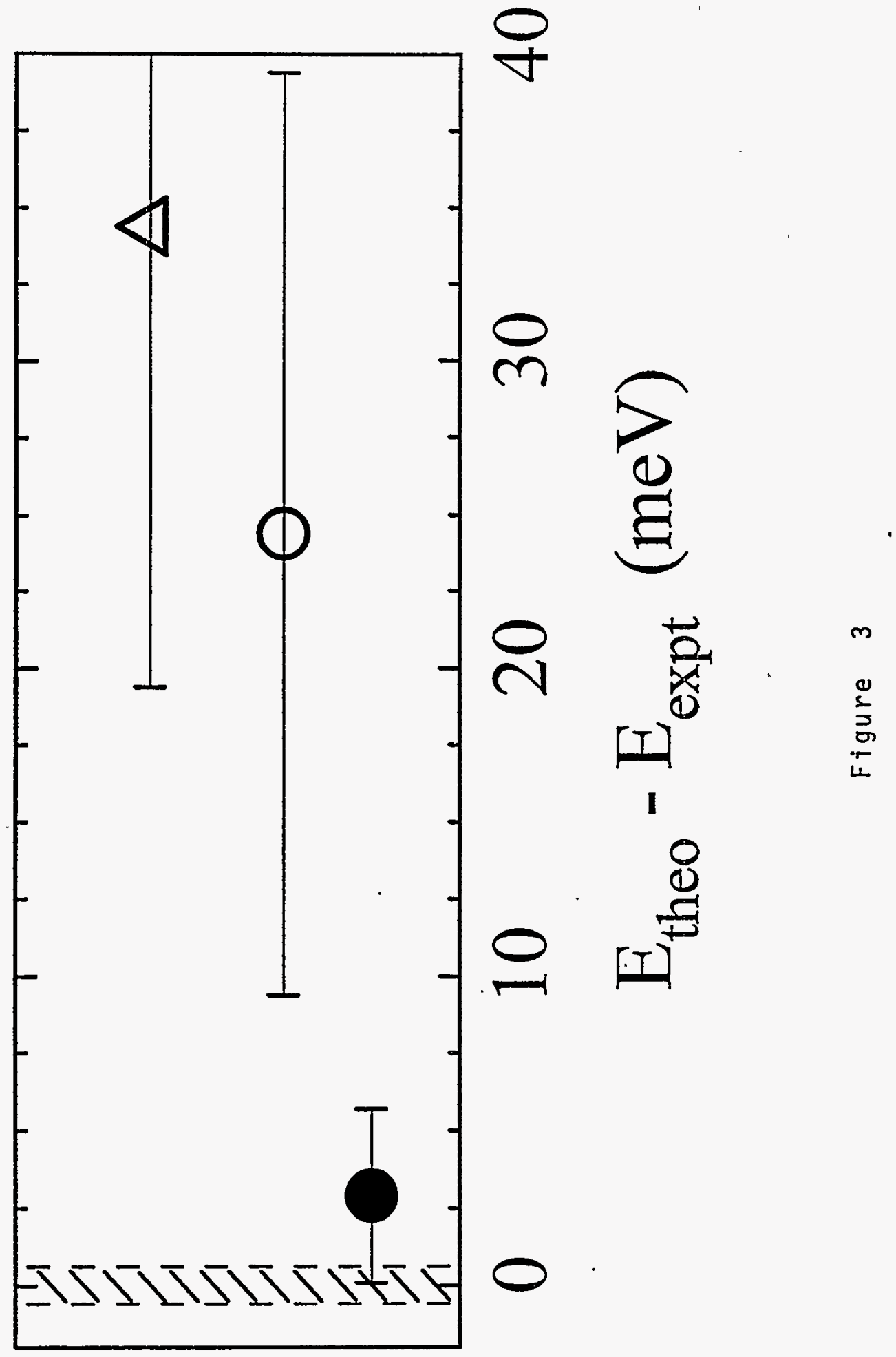




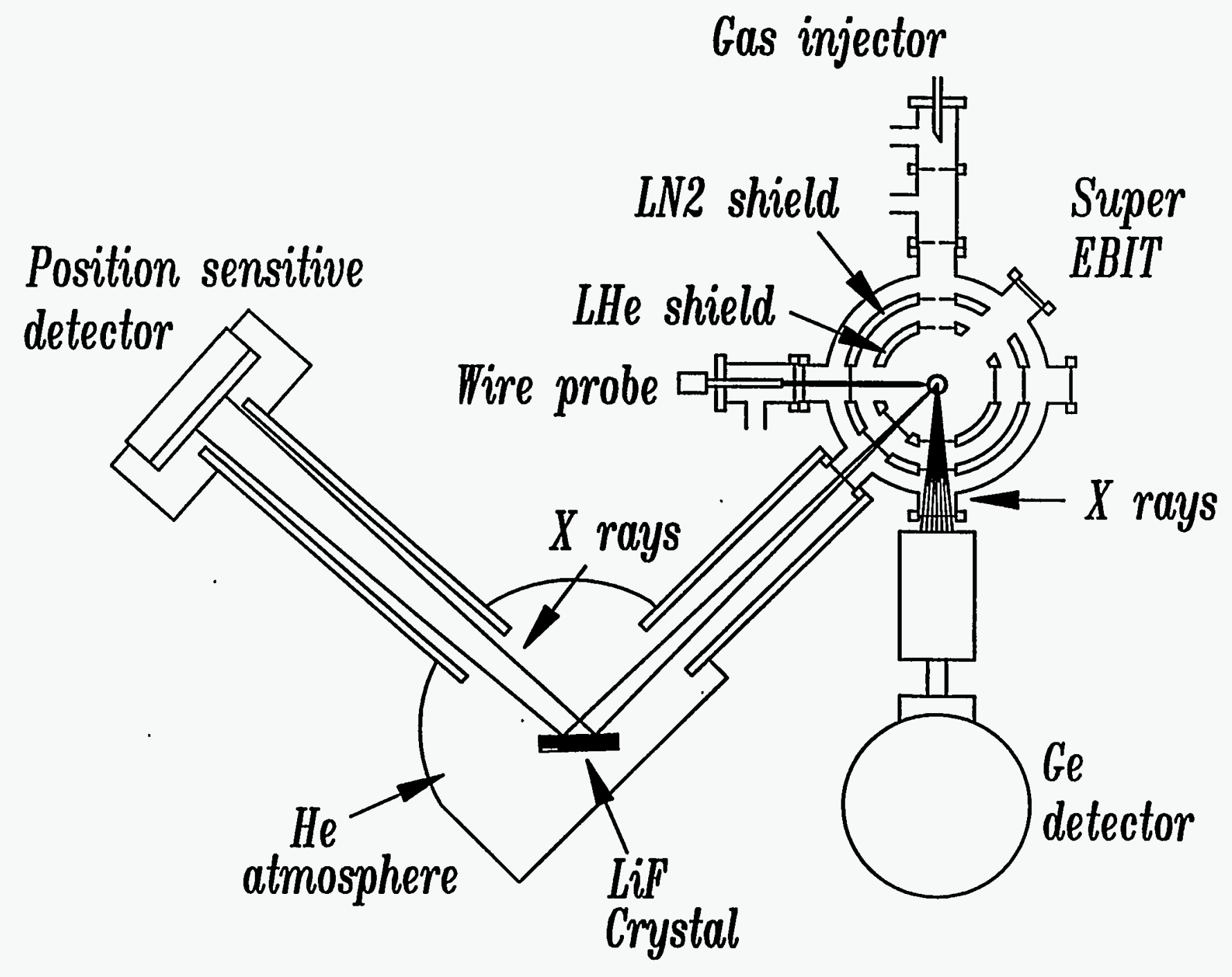

Figure 4 

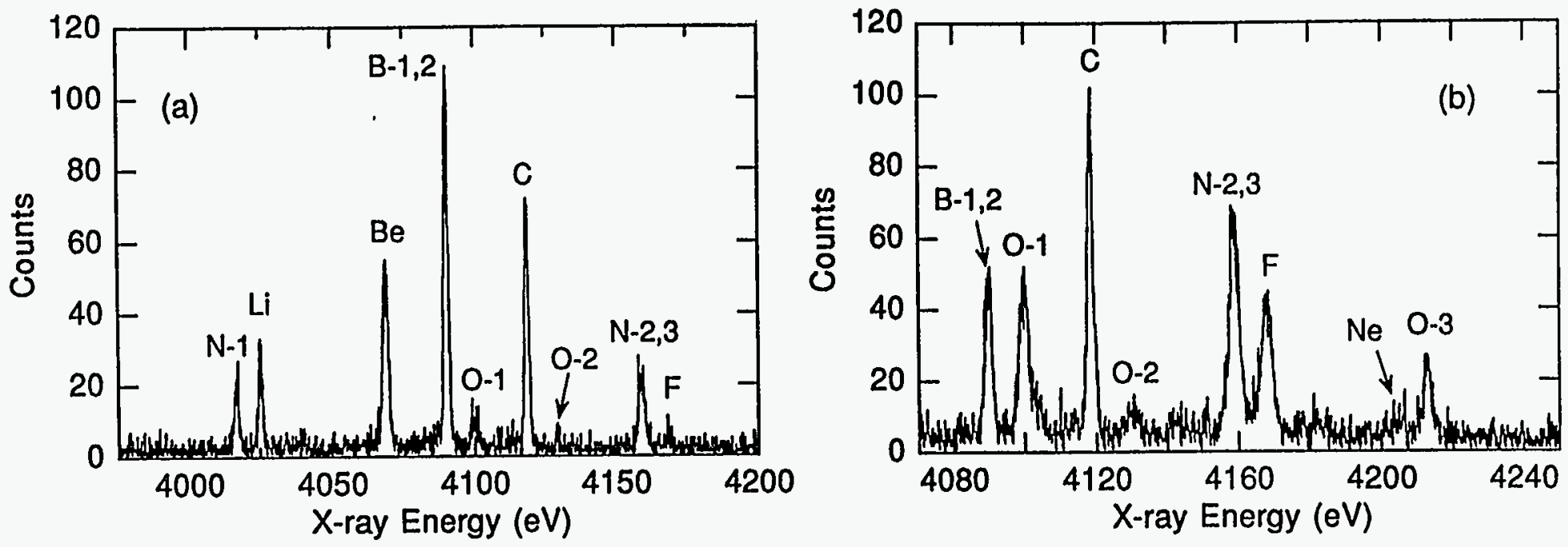

Figure 5 


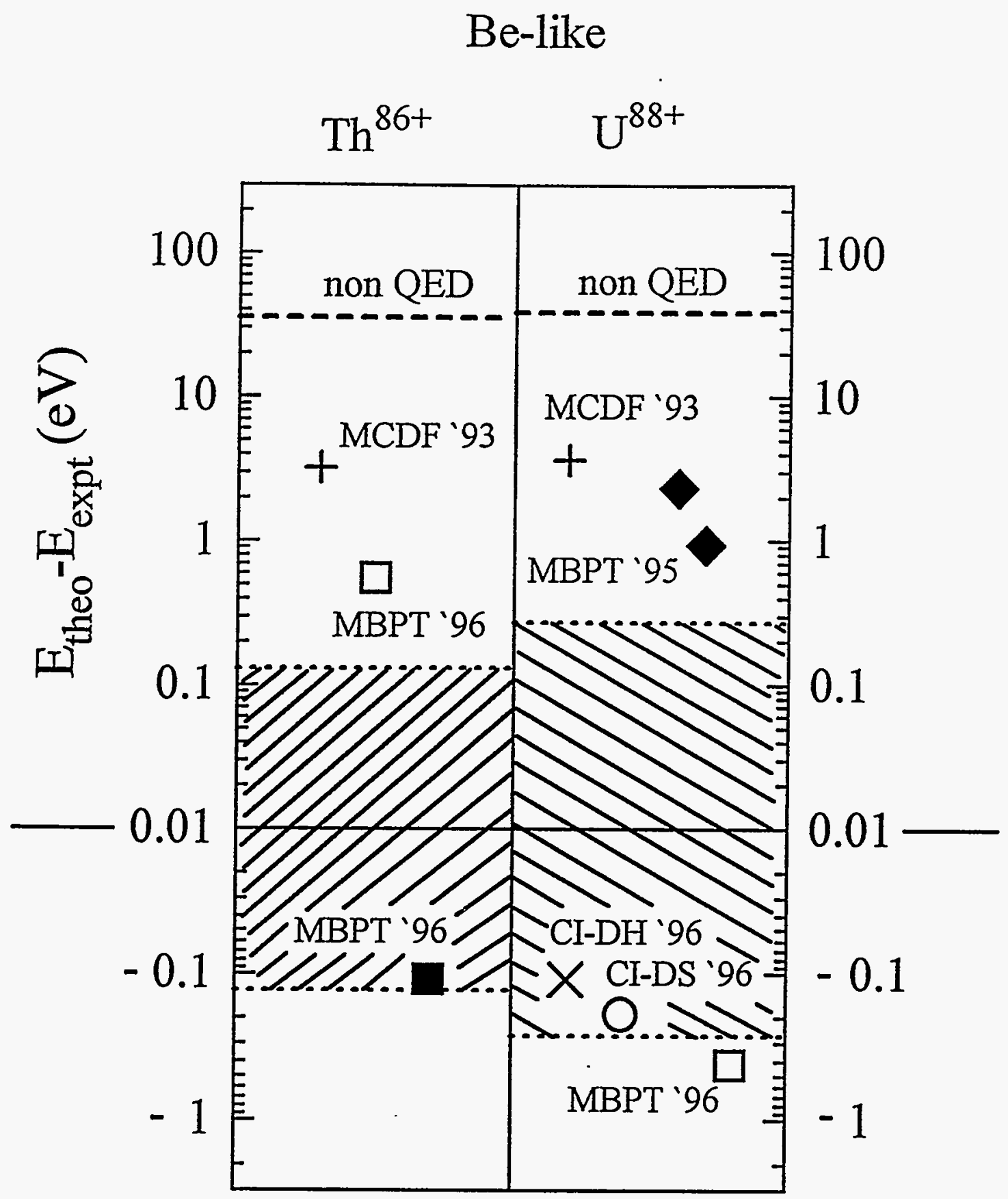

Figure 6 

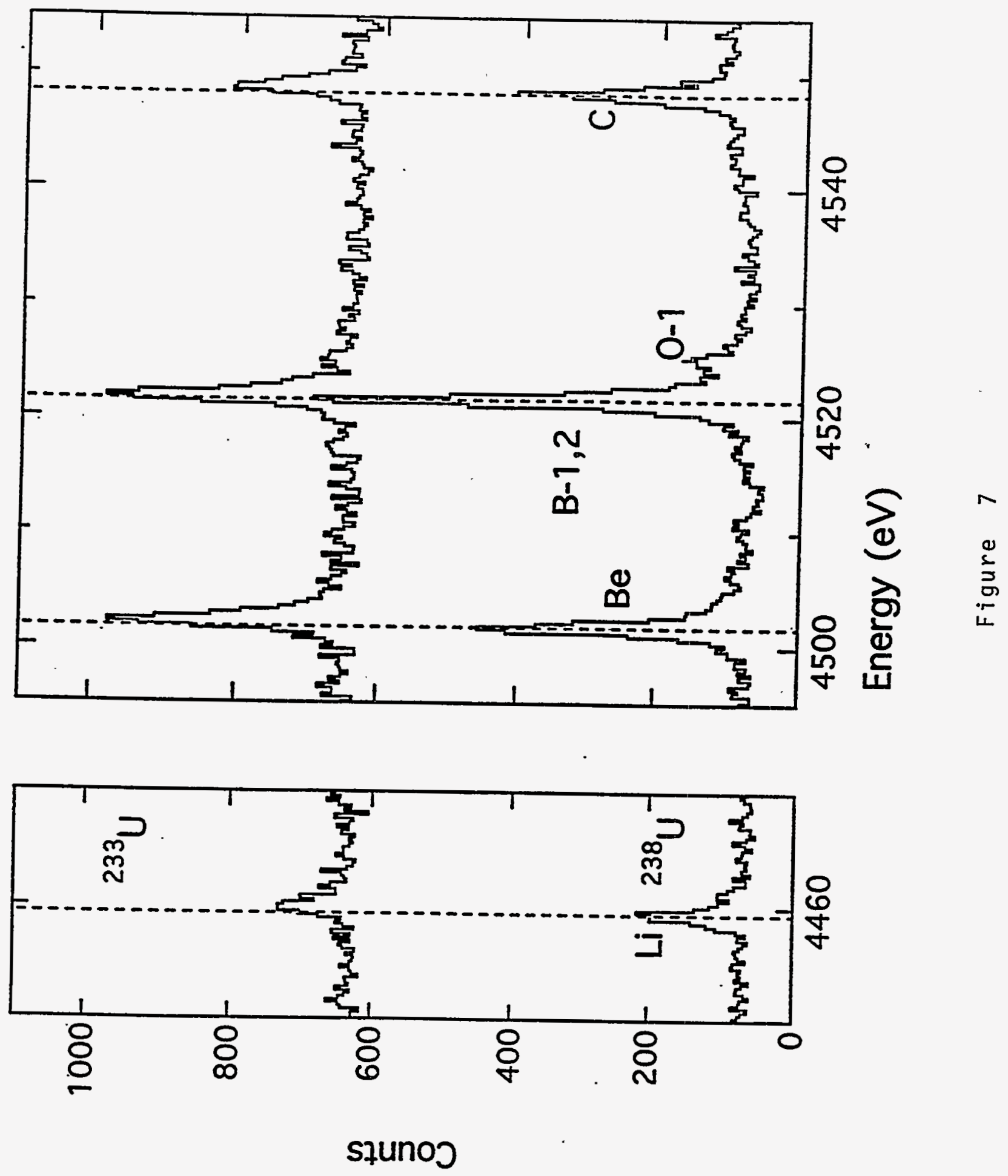


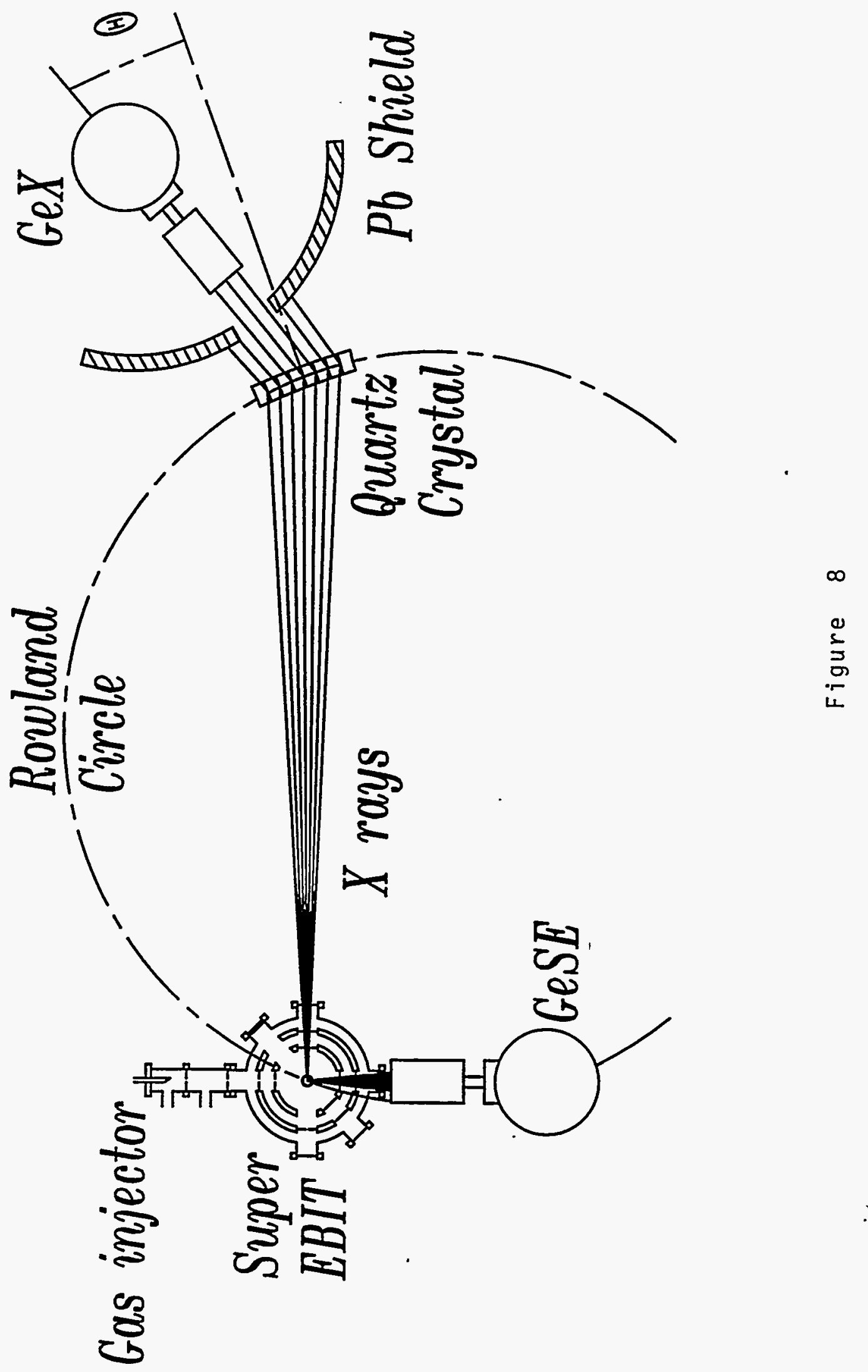


He-like $\mathrm{Xe}^{52+}$

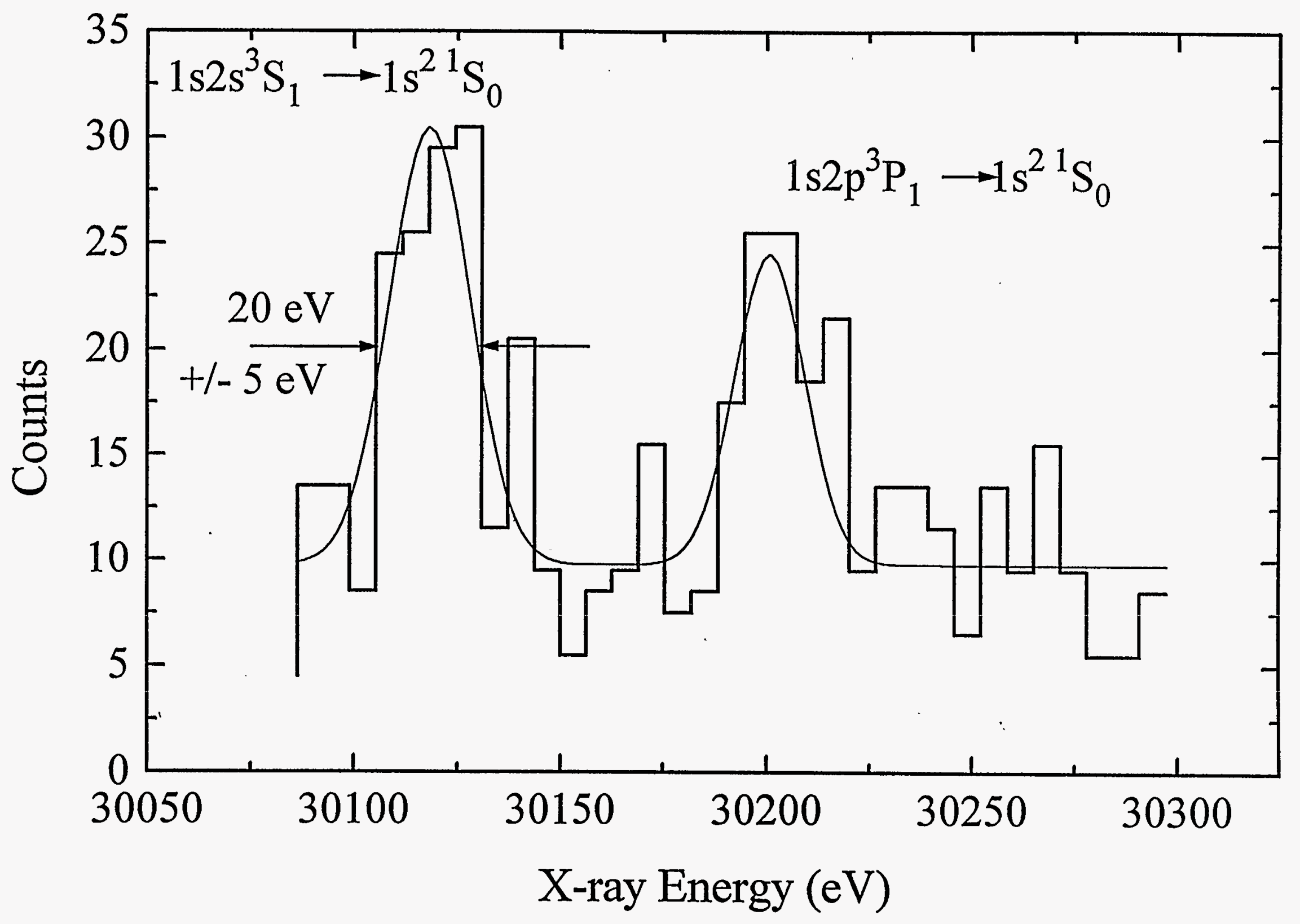

Figure 9 